\title{
Robust implementation of contact under friction and large sliding with the eXtended finite element method
}

\author{
Maximilien Siavelis* — Patrick Massin** — Martin L.E. Guiton* \\ Sylvain Mazet** — Nicolas Moës*** \\ *IFP Geology-Geochemistry-Geophysics \\ 1 à 4 avenue de Bois-Préau, F-92852 Rueil-Malmaison cedex \\ \{maximilien.siavelis,martin.guiton\}@ifp.fr \\ ** LaMSID, EDF R\&D UMR EDF/CNRS 2832 \\ 1 avenue du général de Gaulle, F-92141 Clamart cedex \\ \{patrick.massin,sylvain_externe.mazet\}@edf.fr \\ *** GeM, École Centrale de Nantes/Université de Nantes UMR CNRS \\ 6183, 1 rue de la Noé, F-44321 Nantes cedex 3 \\ nicolas.moes@ec-nantes.fr
}

\begin{abstract}
An X-FEM formulation is proposed for the case of large sliding frictional interfaces. A continuous augmented lagrangian framework is adopted for contact and friction. We provide an algorithm for the selection of an appropriate discrete space for the lagrange multipliers, accounting for the transition between contact and free zones, and also between sliding and adherent zones. A 3D numerical test is realized with Code_Aster free software for the compression of a cylinder cut along a radial section and shows the ability of the model to capture such transitions.

RÉSUMÉ. Une formulation d'X-FEM est proposée pour le cas d'une interface frottante avec grands glissements. Une formulation continue avec lagrangien augmenté décrit le contact et le frottement. Nous détaillons un algorithme qui permet de sélectionner un bon espace des multiplicateurs de lagrange, en tenant compte de la transition entre le contact et le décollement, et de celle entre le glissement et l'adhérence. Un cas numérique $3 D$ de compression d'un cylindre découpé radialement est réalisé avec le logiciel libre Code_Aster et montre l'aptitude de la méthode à capturer de telles transitions.

KEYWORDS: X-FEM, frictional contact, large sliding, 3D, LBB, augmented lagrangian.

MOTS-CLÉS : X-FEM, contact frottant, grands glissements, 3D, LBB, lagrangien augmenté.
\end{abstract}

DOI:10.3166/EJCM.19.189-203 (C) 2010 Lavoisier, Paris

EJCM - 19/2010. Giens 2009, pages 189 to 203 


\section{Introduction}

Building 3D meshes for complex industrial structures is difficult, especially when the mesh has to conform complex geometries of discontinuity surfaces. In this work we are interested in the eXtended Finite Element Method (X-FEM) witch represents a discontinuity thanks to an enrichment of the nodal degrees of freedom (d.o.f.) and thus facilitates the model design (Moës et al., 1999). We focus on the case of a frictional interface.

Several approaches have been proposed to take into account contact and friction in a small sliding context, with penalty (Dolbow et al., 2001; Khoei et al., 2006; Liu et al., 2008) or with Lagrangian regularization techniques (Géniaut et al., 2007; Béchet et al., 2009). The extension to large sliding was developed in (Nistor et al., 2009), by considering that each integration of a contact contribution is associated to a special contact element based on a master slave approach. The difference with FEM contact elements is that slave and master elements are no more constituted by elements discretizing the interface but are built with enriched bulk elements. It is then possible to update the master-slave association with sliding evolution, within a fixed point algorithm.

In this paper we present the extension of Nistor's approach to the frictional case. The mixed displacement-pressure formulation used is based on a variational formulation from (Ben Dhia et al., 2002) which is based on an augmented lagrangian and solved by a Generalized Newton algorithm as proposed by (Alart et al., 1991). As it was discussed in (Babus̃ka, 1973), for mixed methods, particular attention should be paid to the choice of the discrete space of dual unknowns with respect to the displacement space. The latter should typically not be less than the former, if one wants to satisfy the LBB condition (Brezzi et al., 1991) which ensures the existence and uniqueness of the solution. A way to recover this condition is to enrich the displacement, for example with bubble interpolating functions, as shown in (Mourad et al., 2007) for the case of Dirichlet conditions imposed on an interface. However, because the classical bubble functions are vanishing on element edges, this strategy shows limited stabilization of the spurious modes when the interface is nearly conform to the elements. We follow here an alternative choice to reduce the dual space, inspired from (Moës $e t$ al., 2006).

In (Géniaut et al., 2007), the contact and friction unknowns are stored at a node or at the middle of an element edge, to be in association with the intersection of the discontinuity and the mesh topology. The corresponding interpolation functions are thus defined along segments which discretize the interface. To satisfy the LBB condition, an algorithm was proposed which selects vital and non-vital edges and imposes equality or linear relations on the contact multiplier of non vital edges with respect to those of the vital edges. Numerical validations, in the sense of (Chapelle et al., 1993), were given in 2D and 3D. It was shown in (Nistor et al., 2009) that this property was still valid for large sliding because the dual unknowns are only stored on the slave part. 
In the present paper, the same algorithm is used, but the contact unknowns are interpolated like the displacements, that is with respect to nodal values on the volume element. The satisfaction of the LBB condition with this construction of the contact and friction lagrangian space was demonstrated in (Béchet et al., 2009) for a mesh composed of triangles.

The content of this paper is as follows. In the first section, the continuous mixed formulation of contact and friction is presented. The second section provides a description of the X-FEM spatial discretization, emphasizing on the dual space definition. For the case of a mesh composed of tetrahedral in $3 \mathrm{D}$ and triangles in $2 \mathrm{D}$, an additional rule is provided to select the vital edges at the transition between contact and free zones, or between sliding and adherent ones. It helps solving the possible conflict between equality or linear relations linking the multipliers on non vital edges and the corresponding contact or friction status. The case of hexaedra in 3D and quadrilateral in $2 \mathrm{D}$, is also discussed.

The third section provides a numerical example to illustrate the robustness of the method, realized with Code_Aster free software ${ }^{\circledR}$ (http: //www . code-aster.org). A cylinder cut by a frictional interface along a radial section is submitted to a compression leading to a radial transition from an adherent region in the centre, to a sliding region and finally to a non-contacting outer zone.

\section{Mixed continuous formulation of contact and friction}

\subsection{Variational forms}

The displacement can be large, especially along the discontinuity interface, but we keep a small strains and stresses hypothesis, considering elastic materials.

Let us denote $\Omega$ the domain of interest which boundaries are composed of a part $\Gamma_{u}, \Gamma_{t}$ and $\Gamma_{c}$ where conditions are imposed on the displacement $\mathbf{u}$, on the pressure distribution and on the frictional contact, respectively. In this paper, we assume $\Gamma_{c}$ to represent an interface, i.e. a discontinuity that cuts entirely the domain into $\Omega_{1}$ and $\Omega_{2}$, with interfacial boundaries $\Gamma_{c 1}$ and $\Gamma_{c 2}$, respectively. Considering the inward normal $\mathbf{n}$ to $\Omega_{2}$, we can separate the contact friction force $\mathbf{r}$ that $\Omega_{2}$ applies to $\Omega_{1}$ into its normal part $\lambda$ and its tangential part $\mathbf{r}_{\tau}$ as follows:

$$
\mathbf{r}=\lambda \mathbf{n}+\mathbf{r}_{\tau}
$$

Considering arbitrarily $\Gamma_{c 1}$ as a reference surface called "slave", and $\Gamma_{c 2}$ as its corresponding "master" surface, we introduce the normal distance between a slave point $\mathbf{x}^{1}$ and its projection $\overline{\mathbf{x}}^{1}$ on $\Gamma_{c 2}$ :

$$
d_{n}=\left(\mathbf{x}^{1}-\overline{\mathbf{x}}^{\mathbf{1}}\right) \cdot \mathbf{n} .
$$


The Signorini contact condition reads:

$$
\lambda \leq 0, d_{n} \leq 0, \lambda d_{n}=0
$$

The augmented lagrangian regularization is achieved with the augmented multiplier:

$$
g_{n}=\lambda-\rho_{n} d_{n},
$$

where $\lambda$ is the usual Lagrange multiplier for contact and $\rho_{n}$ is a positive scalar homogeneous to a pressure over a displacement.

We also introduce $\chi$, the $\mathbb{R}^{-}$indicator function to obtain from equation [3]:

$$
\lambda-\chi\left(g_{n}\right) g_{n}=0 .
$$

We then introduce the tangential relative velocity as:

$$
\mathbf{v}_{\tau}=(\mathbb{I}-\mathbf{n} \otimes \mathbf{n}) \cdot \frac{\partial\left(\mathbf{u}\left(\mathbf{x}^{\mathbf{1}}\right)-\mathbf{u}\left(\overline{\mathbf{x}}^{\mathbf{1}}\right)\right)}{\partial \mathbf{t}}
$$

where the left second order tensor is the operator of projection on the plane tangent to $\Gamma_{c 2}$ at $\overline{\mathbf{x}}^{1}$. Using $\mu$ the friction coefficient, we define the cohesionless Coulomb's law as:

$$
\mathbf{r}_{\tau}=\mu \lambda \boldsymbol{\Lambda} \text { with }\left\{\begin{array}{clc}
\|\boldsymbol{\Lambda}\|=0 & \text { if } & d_{n}<0, \\
\|\boldsymbol{\Lambda}\| \in] 0,1[ & \text { if } & d_{n}=0 \text { and }\left\|\mathbf{v}_{\tau}\right\|=\mathbf{0}, \\
\boldsymbol{\Lambda}=\frac{v_{\tau}}{\left\|\mathbf{v}_{\tau}\right\|} & \text { if } & d_{n}=0 \text { and }\left\|\mathbf{v}_{\tau}\right\|>\mathbf{0} .
\end{array}\right.
$$

As for contact, an augmented regularization is used with the augmented semimultiplier:

$$
\mathbf{g}_{\tau}=\boldsymbol{\Lambda}+\rho_{\tau} \mathbf{v}_{\tau},
$$

with $\boldsymbol{\Lambda}$ the usual semi-multiplier vector for friction and $\rho_{\tau}$ a positive scalar.

We also introduce $\mathbf{P}_{B(0,1)}$ the projection operator on the unit ball:

$$
\mathbf{P}_{B(0,1)}(\mathbf{x})=\left\{\begin{array}{l}
\mathbf{x} \text { if }\|\mathbf{x}\|<\mathbf{1} \\
\frac{\mathbf{x}}{\|\mathbf{x}\|} \text { if }\|\mathbf{x}\| \geq \mathbf{1}
\end{array}\right.
$$

to simplify the friction condition [7] into:

$$
\mathbf{\Lambda}-\chi\left(g_{n}\right) \mathbf{P}_{B(0,1)}\left(\mathbf{g}_{\tau}\right)=0
$$




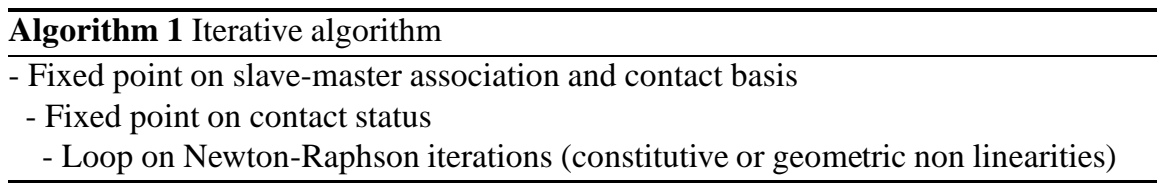

Taking into account the augmented lagrangian allows to control the conditioning of the system (Alart et al., 1991). The contact and tangential reactions being represented by $\chi\left(g_{n}\right) g_{n}$ and $\mu \chi\left(g_{n}\right) g_{n} \mathbf{P}_{B(0,1)}\left(\mathbf{g}_{\tau}\right)$, respectively, the variational form of the equilibrium, for any variation of displacement $\mathbf{u}^{*}$ reads:

$$
\begin{gathered}
\int_{\Omega} \boldsymbol{\sigma}(\boldsymbol{\epsilon}(\mathbf{u})): \boldsymbol{\epsilon}\left(\mathbf{u}^{*}\right) d \Omega-\int_{\Gamma_{c}} \chi\left(g_{n}\right) g_{n} \mathbf{n} \cdot \llbracket \mathbf{u}^{*} \rrbracket d \Gamma \\
-\int_{\Gamma_{c}} \mu \chi\left(g_{n}\right) g_{n} \mathbf{P}_{B(0,1)}\left(\mathbf{g}_{\tau}\right) \cdot(\mathbb{I}-\mathbf{n} \otimes \mathbf{n}) \cdot \llbracket \mathbf{u}^{*} \rrbracket d \Gamma=L_{\text {meca }}\left(\mathbf{u}^{*}\right),
\end{gathered}
$$

where $\llbracket \mathbf{u} \rrbracket=\mathbf{u}\left(\mathbf{x}^{\mathbf{1}}\right)-\mathbf{u}\left(\overline{\mathbf{x}}^{\mathbf{1}}\right)$. The first term is the contribution from internal work, denoted as the double product between the Cauchy stress tensor $\boldsymbol{\sigma}$ and the virtual strain $\boldsymbol{\epsilon}$. The term $L_{\text {meca }}$ represents the possible contribution from body force and applied pressure on $\Gamma_{t}$ which are not of interest in this paper. A difference with (Ben Dhia et $a l ., 2002)$ can be noted in the dissipative frictional part of equation [11], $g_{n}$ replacing $\lambda$ because we have chosen to remove the fixed point algorithm on the friction threshold (see 2.2).

Following the mixed formulation of (Ben Dhia et al., 2002), we also consider the variational forms for the contact [5] and friction [10] conditions, for all variations $\lambda^{*}$ and $\mathbf{\Lambda}^{*}$ as:

$$
\begin{aligned}
\int_{\Gamma_{c}}-\frac{1}{\rho_{n}}\left(\lambda-\chi\left(g_{n}\right) g_{n}\right) \lambda^{*} d \Gamma & =0 \\
\int_{\Gamma_{c}} \frac{1}{\rho_{\tau}}\left(\mathbf{\Lambda}-\chi\left(g_{n}\right) \mathbf{P}_{B(0,1)}\left(\mathbf{g}_{\tau}\right)\right) \cdot \mathbf{\Lambda}^{*} d \Gamma & =0 .
\end{aligned}
$$

\subsection{Iterative algorithm for non linearities}

The iterative algorithm to take into account non linearities is shown in Algorithm 1. The external loop is a fixed point on the change of slave-master contact association during large sliding. Note that the unit normal vector $\mathbf{n}$ which carries the normal contact reaction is fixed during an iteration of this loop. The second inner loop is a fixed point on the contact status, i.e. $\chi\left(g_{n}\right)$, as in (Dumont, 2001). Finally, the non linearities coming from the friction law are solved by linearizing the terms of equations [11],[12] and [13] in the tangent operators of the Newton-Raphson loop. Let us 
consider a Newton iteration, with $[\mathbf{u}, \lambda, \boldsymbol{\Lambda}]$ the initial given fields. Noting $[\delta \mathbf{u}, \delta \lambda, \delta \boldsymbol{\Lambda}]$ the incremental variations, we obtain:

$$
\begin{array}{r}
\int_{\Omega} \boldsymbol{\epsilon}(\delta \mathbf{u}): \frac{\partial \boldsymbol{\sigma}}{\partial \boldsymbol{\epsilon}}: \boldsymbol{\epsilon}\left(\mathbf{u}^{*}\right) d \Omega-\int_{\Gamma_{c}} \chi \delta \lambda\left[\mathbf{u}^{*}\right]_{n} d \Gamma+\int_{\Gamma_{c}} \chi \rho_{n}[\delta \mathbf{u}]_{\mathbf{n}}\left[\mathbf{u}^{*}\right]_{\mathbf{n}} d \Gamma \\
-\int_{\Gamma_{c}} \chi \mu \delta \lambda \mathbf{P}_{B}\left(\mathbf{g}_{\tau}\right) \cdot\left[\mathbf{u}^{*}\right]_{\tau} d \Gamma+\int_{\Gamma_{c}} \chi \mu \rho_{n}[\delta \mathbf{u}]_{\mathbf{n}} \mathbf{P}_{\mathbf{B}}\left(\mathbf{g}_{\tau}\right) \cdot\left[\mathbf{u}^{*}\right]_{\tau} d \Gamma \\
-\int_{\Gamma_{c}} \chi \mu g_{n} K\left(\mathbf{g}_{\tau}\right) \delta \boldsymbol{\Lambda}\left[\mathbf{u}^{*}\right]_{\tau} d \Gamma-\int_{\Gamma_{c}} \chi \mu g_{n} \rho_{\tau} K\left(\mathbf{g}_{\tau}\right)[\delta \mathbf{u}]_{\tau}\left[\mathbf{u}^{*}\right]_{\tau} d \Gamma \\
=-\int_{\Omega} \boldsymbol{\sigma}(\boldsymbol{\epsilon}(\mathbf{u})): \boldsymbol{\epsilon}\left(\mathbf{u}^{*}\right) d \Omega+L_{m e c a}\left(\mathbf{u}^{*}\right) \\
+\int_{\Gamma_{c}} \chi g_{n}\left[\mathbf{u}^{*}\right]_{n} d \Gamma+\int_{\Gamma_{c}} \chi \mu g_{n} \mathbf{P}_{B}\left(\mathbf{g}_{\tau}\right) \cdot\left[\mathbf{u}^{*}\right]_{\tau} d \Gamma \\
-\int_{\Gamma_{c}} \frac{1-\chi}{\rho_{n}} \delta \lambda \lambda^{*} d \Gamma-\int_{\Gamma_{c}} \chi[\delta \mathbf{u}]_{\mathbf{n}} \lambda^{*} d \Gamma=\int_{\Gamma_{c}} \frac{1}{\rho_{n}}\left(\lambda-\chi g_{n}\right) \lambda^{*} d \Gamma \\
+\int_{\Gamma_{c}} \frac{1}{\rho_{\tau}}\left(\mathbb{I I}-\chi \mathrm{K}\left(\mathrm{g}_{\tau}\right)\right) \delta \boldsymbol{\Lambda} \cdot \boldsymbol{\Lambda}^{*} d \Gamma-\int_{\Gamma_{c}} \chi K\left(g_{\tau}\right)[\delta \mathbf{u}]_{\tau} \cdot \boldsymbol{\Lambda}^{*} d \Gamma \\
=-\int_{\Gamma_{c}} \frac{1}{\rho_{\tau}}\left(\boldsymbol{\Lambda}-\chi \mathbf{P}_{B}\left(\mathbf{g}_{\tau}\right)\right) \cdot \boldsymbol{\Lambda}^{*} d \Gamma
\end{array}
$$

with:

$$
\begin{aligned}
& \mathbf{K}(\mathbf{x})=\left\{\begin{array}{c}
\mathbb{I} \text { if }\|\mathbf{x}\|<\mathbf{1}, \\
\frac{1}{\|\mathbf{x}\|}\left(\mathbb{I}-\frac{\mathbf{x} \otimes \mathbf{x}}{\|\mathbf{x}\|^{2}}\right) \text { if }\|\mathbf{x}\| \geq \mathbf{1},
\end{array}\right. \\
& {[\mathbf{x}]_{\mathbf{n}}=\mathbf{n} \cdot \llbracket \mathbf{x} \rrbracket \quad \text { and } \quad[\mathbf{x}]_{\tau}=(\mathbb{I}-\mathbf{n} \otimes \mathbf{n}) \cdot \llbracket \mathbf{x} \rrbracket .}
\end{aligned}
$$

\section{X-FEM discretization and LBB condition}

\subsection{Spatial discretization}

Because the contact method of (Ben Dhia et al., 2002) is a continuous formulation, its adaptation to X-FEM is facilitated. Likewise a FEM approach, the slave contact surface provides a discretization for the numerical quadrature of contact and friction integrals in equations [14], [15] and [16]. The main difference is that the slave surface containing the integration points and also the master one are no more defined by element boundaries, but are interpolated from the intersection points between the interface and the mesh as proposed in (Nistor et al., 2009). For sake of simplicity, we restrict our discussion to the case where the surfaces are discretized with linear segment in $2 \mathrm{D}$, triangles in $3 \mathrm{D}$. 
For each integration point, its projection on the master surface [2] is used to build a contact element composed of the slave element nodes and the master ones. During the pairing, the reference coordinates of the integration point in the bulk slave element and the reference coordinates of its projection in the bulk master element are needed. Using the corresponding bulk shape functions $\phi^{m}$ and $\phi^{s}$ in these two elements, respectively, the slave and master displacements $\mathbf{u}^{\mathbf{s}}$ and $\mathbf{u}^{\mathbf{m}}$ read:

$$
\mathbf{u}^{\mathbf{s}}=\sum_{\mathbf{j}=\mathbf{1}}^{\mathbf{n}_{\mathbf{s}}} \phi_{\mathbf{j}}^{\mathbf{s}}\left(\mathbf{u}_{\mathbf{j}}^{\mathbf{s}}-\mathbf{a}_{\mathbf{j}}^{\mathbf{s}}\right) \text { and } \mathbf{u}^{\mathbf{m}}=\sum_{\mathbf{j}=\mathbf{1}}^{\mathbf{n}_{\mathbf{m}}} \phi_{\mathbf{j}}^{\mathbf{m}}\left(\mathbf{u}_{\mathbf{j}}^{\mathbf{m}}+\mathbf{a}_{\mathbf{j}}^{\mathbf{m}}\right),
$$

where a denotes the additional nodal variables representing the interface discontinuity. Note that the difference of sign preceding a in the master and slave part corresponds to the use of a generalized Heaviside function to describe the discontinuity.

Considering equation [19] and introducing the initial spatial coordinate $\mathbf{X}$, we can discretise [2] and [6] for a given numerical time step $\Delta t$ as:

$$
\begin{aligned}
& d_{n}=\left(\sum_{j=1}^{n_{s}} \phi_{j}^{s}\left(\mathbf{X}_{\mathbf{j}}^{\mathbf{s}}+\mathbf{u}_{\mathbf{j}}^{\mathbf{s}}-\mathbf{a}_{\mathbf{j}}^{\mathbf{s}}\right)-\sum_{\mathbf{j}=\mathbf{1}}^{\mathbf{n}_{\mathbf{m}}} \phi_{\mathbf{j}}^{\mathbf{m}}\left(\mathbf{X}_{\mathbf{j}}^{\mathbf{m}}+\mathbf{u}_{\mathbf{j}}^{\mathbf{m}}+\mathbf{a}_{\mathbf{j}}^{\mathbf{m}}\right)\right) \cdot \mathbf{n}, \\
& \mathbf{v}_{\tau}=\frac{(\mathbb{I}-\mathbf{n} \otimes \mathbf{n})}{\Delta \mathbf{t}} \cdot\left(\sum_{\mathbf{j}=\mathbf{1}}^{\mathbf{n}_{\mathbf{s}}} \phi_{\mathbf{j}}^{\mathbf{s}} \boldsymbol{\Delta}\left(\mathbf{u}_{\mathbf{j}}^{\mathbf{s}}-\mathbf{a}_{\mathbf{j}}^{\mathbf{s}}\right)-\sum_{\mathbf{j}=\mathbf{1}}^{\mathbf{n}_{\mathbf{m}}} \phi_{\mathbf{j}}^{\mathbf{m}} \boldsymbol{\Delta}\left(\mathbf{u}_{\mathbf{j}}^{\mathbf{m}}+\mathbf{a}_{\mathbf{j}}^{\mathbf{m}}\right)\right) .
\end{aligned}
$$

The contact unknowns are only defined on the slave part and we choose to interpolate the contact and friction forces from the nodes of the bulk element. The contact lagrangian and friction semi-lagrangian at the integration point then read:

$$
\lambda=\sum_{j=1}^{n_{s}} \phi_{j}^{s} \lambda_{j} \text { and } \mathbf{\Lambda}=\sum_{j=1}^{n_{s}} \phi_{j}^{s} \boldsymbol{\Lambda}_{j} .
$$

Using equations [20] and [21], the linearized formulation [14],[15] and [16] can be expressed in a matrix form as follows, omitting the iteration indexes for sake of clarity:

$$
\left[\begin{array}{ccc}
\mathbf{K}_{\mathbf{u}}+\mathbf{A}_{\mathbf{u}}+\mathbf{B}_{\mathbf{u}} & \mathbf{A} & \mathbf{B} \\
\mathbf{D} & \mathbf{C} & \mathbf{0} \\
\mathbf{E} & \mathbf{0} & \mathbf{F}
\end{array}\right]\left(\begin{array}{c}
\delta \mathbf{u} \\
\delta \lambda \\
\delta \boldsymbol{\Lambda}
\end{array}\right)=\left(\begin{array}{c}
\mathbf{L}_{\mathbf{u}}+\mathbf{L}_{\lambda}^{1}+\mathbf{L}_{\boldsymbol{\Lambda}}^{1} \\
\mathbf{L}_{\lambda}^{2} \\
\mathbf{L}_{\boldsymbol{\Lambda}}^{2}
\end{array}\right)
$$

where the first, second and third rows comes from the discretization of equations [14], [15] and [16], respectively. $\mathbf{K}_{\mathbf{u}}, \mathbf{A}_{\mathbf{u}}$ and $\mathbf{B}_{\mathbf{u}}$ are the mechanical, the augmented contact and the augmented friction stiffness matrices, respectively. The matrices $\mathbf{A}, \mathbf{B}$ and the second members $\mathbf{L}_{\lambda}^{1}, \mathbf{L}_{\Lambda}^{1}$ come from the contact and friction forces, respectively. Note that the matrix $\mathbf{B}_{\mathbf{u}}$ is not symmetric and matrices $\mathbf{D}$ and $\mathbf{E}$ are not the transposes of $\mathbf{A}$ and $\mathbf{B}$, respectively, due to the linearization of the friction terms, while a fixed point method on the threshold was used in (Ben Dhia et al., 2002). Despite the fact that the global matrix looses symmetry when we consider friction, this enables to synchronize the changes for the friction augmented semi-multiplier with respect to the contact one, as in (Alart et al., 1991), thus optimizing the global convergence and limiting the occurrence of oscillation pathologies on the friction status. In other words, a Newton iteration state always stays inside the Coulomb's cone [7]. 


\subsection{Lagrangian space reduction}

\subsubsection{Introduction of vital edges}

With X-FEM, a naive linear P1-P1 interpolation choice for the displacement and contact friction spaces, respectively (i.e. with a contact d.o.f located at a cut edge) is not stable and imply oscillations (Géniaut et al., 2005) that we can see on Figure 3 presented hereafter. Our chosen space is a more appropriate one based on the algorithm presented in (Moës et al., 2006). The basic idea of this algorithm is to define the score of each node as the number of its connected cut edges, while the score of a cut edge is given by the minimum score of its contributing nodes. Then we select cut edges with the highest scores that do not have nodes in common: in case of a multiple choice we select randomly one of the possible edges. At each step, we re-compute the node scores without the previously selected edges. The algorithm stop when all the remaining cut edges have a score equal to one. If the minimum score is reached on one edge, this edge is vital. If the minimum score is reached on several edges, their multipliers are imposed to be equal, and only one of these edges is chosen to be vital. Finally, one group of edges with equal multipliers at the end of the algorithm is connected by a linear relation to another group of edges sharing another multiplier if both groups were connected initially by a common edge. The d.o.f of the non-vital edges are thus linked to the d.o.f of the vital ones with linear or equality relations. To provide a better approximation of the pressure space, this algorithm was improved in (Géniaut et al., 2007) to maximize the number of linear relations with respect to equality ones. However in $3 \mathrm{D}$ this leads to convergence difficulties such as flip-flop. The problem is illustrated in Figure 1, where two sliding nodes 1 and 2 are linked linearly to a third one. In that case, node 3 cannot be sliding because $\left\|\boldsymbol{\Lambda}_{3}\right\|<1$. To avoid this problem the linear relation must link the norms of the friction semi-multipliers (left picture), but the relation becomes non-linear for its components.

In (Géniaut et al., 2007), only a numerical validation for the LBB condition as in (Chapelle et al., 1993) was presented. For that reason, we changed the algorithm for the more recent one of (Béchet et al., 2009) for which an analytical proof of the LBB condition satisfaction was given for 2D triangular meshes. In the first approach, the contact pressures were interpolated from unknowns located at cut edges. In the second approach their interpolation is realized with respect to the nodal values [21] and for each vital edge, an equality relation is linking the contact d.o.f of the two vital nodes composing it. Non vital nodes have then to be linked to the neighbouring vital ones by a chosen linear relation. Moreover due to the difficulty of imposing non-linear relations on the semi-multiplier components of $\boldsymbol{\Lambda}$ in 3D corresponding to a linear relation on its norm, we decided to link it to only one of its vital connected node by an equality relation thus lowering the order of the interpolation which satisfies implicitly the LBB condition. 


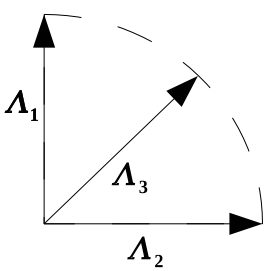

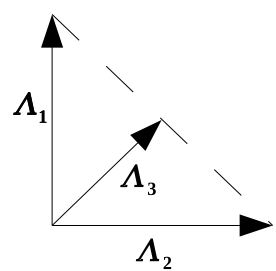

b

Figure 1. Linear relation for $\boldsymbol{\Lambda}$, set on the norm (a) or on the component (b)

\subsubsection{Integration scheme for contact and friction laws}

Because we use linear (for tetrahedra) or bilinear (for hexaedra) interpolation for the displacement, we have chosen a nodal quadrature scheme to integrate the contact friction forces in equation [14]. However due to the algorithm reducing the Lagrangian space, we can lower the numerical quadrature in the contact and friction laws by keeping only integration points at vital edges. This modified integration scheme becomes mandatory in case of transition between free and contact zones, or adherent and sliding ones. Indeed, in the 2D example of Figure 2, assume edge 1 and 3 are vital while edge 2 is not:

$$
\lambda_{1}=\lambda_{2} \quad \text { and } \quad \lambda_{3}=\lambda_{4},
$$

and consider points 1 and 2 are not in contact:

$$
\left(\lambda_{1}+\lambda_{2}\right) / 2=0 \quad \text { and } \quad\left(\lambda_{2}+\lambda_{3}\right) / 2=0 .
$$

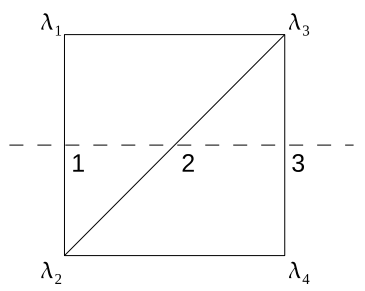

Figure 2. Status and reduced space: point 3 is in contact while 1 and 2 are free

This implies that the contact normal reaction at point 3 is equal to zero and triggers compensation in the neighbouring locations with excessive normal contact reaction. The modified integration scheme we propose involves no contribution in the contact equation [15] from point 2, which is not on a vital edge, and thus solves this conflict. 
Figure 3 shows the results for a simple $2 \mathrm{~d}$ test with frictional horizontal large sliding of a block overlaid by a top block on which we impose a vertical compression. The mesh is composed of triangles. In that case, the left part of the interface between the two blocks is free while the right one is under contact. On the left picture, we compare a FEM case (circles) with a naive X-FEM case (triangles) and an X-FEM case satisfying the LBB condition (diamonds). The naive approach is marked by strong oscillations along the whole contacting zone. These oscillations are solved with the algorithm reducing the lagrangian space. However, near the limit between contact and free zones, significant oscillations can still be noticed, because of the typical pathology previously described. On the right picture, we compare the FEM case (circles) with the X-FEM case satisfying the LBB (diamonds) and the X-FEM case satisfying the LBB with the modified integration scheme (squares). The latter eliminates the oscillations reported above.
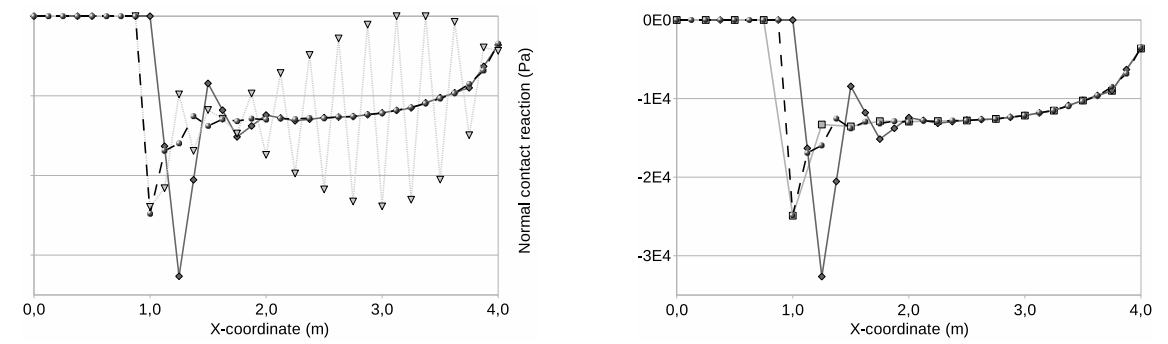

Figure 3. Normal contact reaction vs position along the interface. The response for FEM, naive X-FEM, X-FEM with reduced lagrangian space, and X-FEM with modified integration rule are represented by circles, triangles, diamonds and squares, respectively

The same demonstration can be applied to the case of adherent/sliding transition. Indeed, consider in Figure 2 points 1 and 2 to be sliding (see the third equation of [7]):

$$
\left(\left\|\boldsymbol{\Lambda}_{1}\right\|+\left\|\boldsymbol{\Lambda}_{2}\right\|\right) / 2=1 \quad \text { and } \quad\left(\left\|\boldsymbol{\Lambda}_{2}\right\|+\left\|\boldsymbol{\Lambda}_{3}\right\|\right) / 2=1,
$$

With the help of equation [23] where $\lambda$ is replaced by $\boldsymbol{\Lambda}$, one can notice that point 3 is not allowed to be adherent. We thus propose the same modified integration scheme to solve this problem and do not assemble contributions on non vital edges in the friction equation only [16]. The impact on the global system [22] of the modified integration scheme is very different for contact and friction. For contact, only matrix $\mathrm{C}$ is changed and the global system is still symmetric, while for friction matrices $\mathrm{E}$ and $\mathrm{F}$ are modified leading to another loss of symmetry of the global system. 
Table 1. Vital edge criteria in a group of connected edges

\begin{tabular}{|c|c|}
\hline integration points status on the connected edges & the vital edge must respect: \\
\hline all points are free & no condition \\
\hline there is at least one point in contact & it must contain \\
all the points in contact are sliding & a contacting point \\
\hline there is at least one point in contact & it must contain an \\
there is at least one adherent point & adherent contacting point \\
\hline
\end{tabular}

\subsubsection{Additional criteria to select a vital edge}

When meshes are cut by interfaces, we can obtain groups of connected edges among which we have the choice to select the vital one. This comes frequently for triangle and tetraedra meshes.

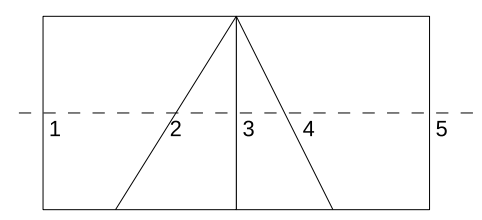

Figure 4. A group of connected edges, edges 1 an 5 are vital, one in [2, 3, 4] is randomly vital

For example in Figure 4, edges 1 and 5 are vital, but the LBB condition algorithm selects randomly a vital edge between 2,3 or 4 . If the integration points on the selected vital edge are not in contact, we can obtain the same kind of conflict as the one described in 3.2.2. In fact, we must avoid a non contacting integration point to impose a zero pressure to the other integration points normally in contact. The same demonstration applies to friction: we must avoid a sliding integration point to impose a norm one value on the semi-lagrangian multipliers of other adherent integration points. Table 1 summarizes the criteria a vital edge in a group must satisfy. Since the contact status can change at each contact iteration and since the friction status can change at each Newton-Raphson iteration, we propose to switch from Algorithm 1 to Algorithm 2 which provides the possibility to change vital edges during the computation.

\subsubsection{Particularities of a hexaedra mesh}

For the case of meshes with quadrilateral or hexaedra cut by an interface, it is possible to have a node not connected to a cut edge inside an element cut by the interface. For example in Figure 5, node 3 is not connected to the other ones. In order to use the nodal interpolation of the pressure on the crack interface, the lagrange multiplier unknowns on this node will be expressed in terms of the multipliers of nodes belonging to edges cut by the interface. For sake of simplicity, we choose to link this 


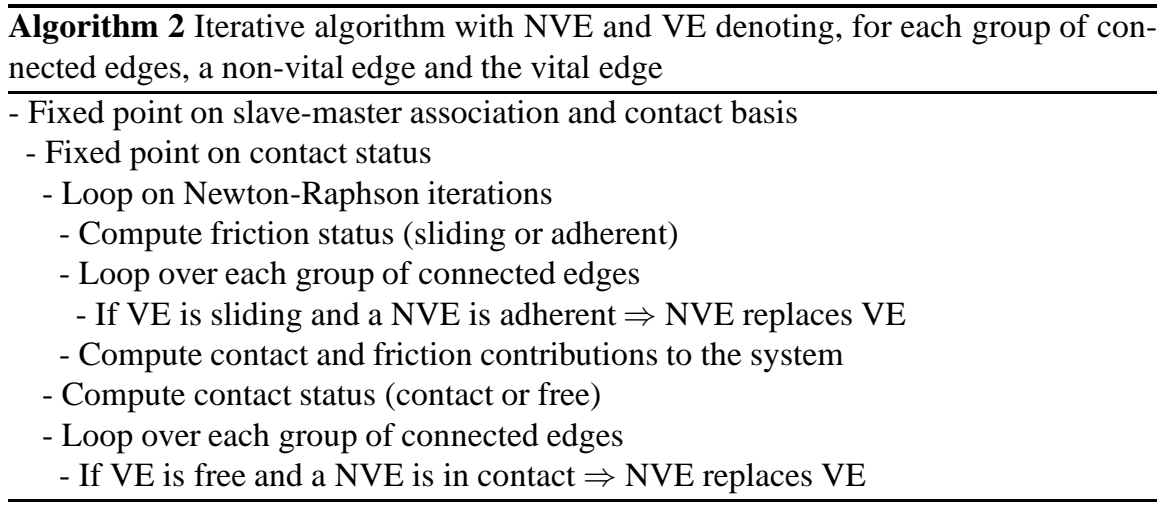

node to its direct neighbours on uncut edges with a linear relation, expressed in this example as:

$$
\lambda_{3}=\left(\lambda_{4}+\lambda_{2}\right) / 2 .
$$

As mentioned in 3.2, the extension of this relation to the semi-multiplier of friction should be done on its norm which leads to a non linear relation on its components in 3D. An alternative is to eliminate the lagrange multipliers of nodes with uncut edges and to modify the contact friction shape function for the other ones in order to assess partition of unity on the element. We propose the following distribution by noting $N_{1}$ and $N_{2}$ the groups of nodes belonging to edges that are cut or not, respectively:

$$
\lambda=\sum_{i \in N_{1}} \tilde{\phi}_{i}^{s} \lambda_{i} \text { with } \tilde{\phi}_{i \in N_{1}}^{s}=\phi_{i}^{s}+\frac{\sum_{j \in N_{2}} \phi_{j}^{s}}{\operatorname{card}\left(N_{1}\right)}
$$

where $\operatorname{card}\left(N_{1}\right)$ is the total number of elements in $N_{1}$.

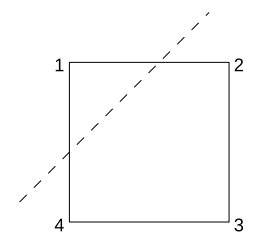

Figure 5. A cut quadrangle, in that case node 3 is not connected to a cut edge 


\section{Numerical example of a 3D cut cylinder under compression}

Let us consider the compression of a linear elastic cylinder with elasticity modulus and Poisson's ratio set to $80 \mathrm{GPa}$ and 0.2 , respectively. This cylinder is cut along a radial section, and is resting on a quasi-rigid substratum (Figure 6). Constant pressures $P_{l}$ of $150 \mathrm{MPa}$ and $P_{t}$ of $50 \mathrm{Mpa}$ are imposed on the lateral and top surfaces, respectively while the lower part is fixed. The friction coefficient is set to 1.0 on the interface.
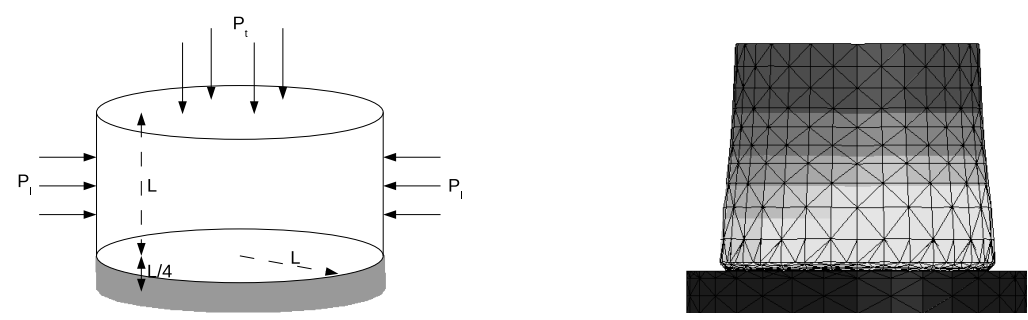

Figure 6. Geometry and boundary conditions are shown on the left with $L=0.04 m$. On the right, the deformed configuration is shown for the tetrahedra case with a radial and vertical amplifications set to 200 and 2000, respectively

Two meshes are considered in the X-FEM cases, with 26880 tetrahedra and 6240 hexaedra, respectively. The deformed configuration for the first mesh is shown in Figure 6. The normal contact reaction at the interface and along a radial profile is given in Figure 7 on the left and right, for the case of the first mesh and the second mesh, respectively. In both cases, we compare the result with respect to that of a FEM model built with 8160 hexaedra and conforming to the interface.

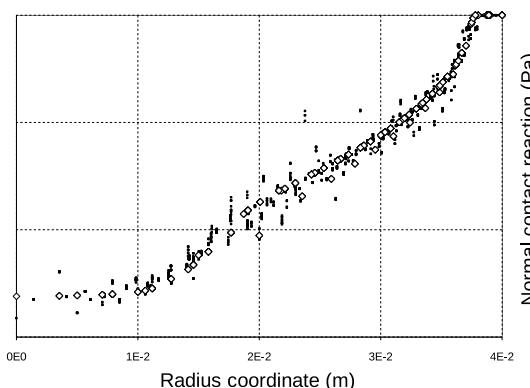

a

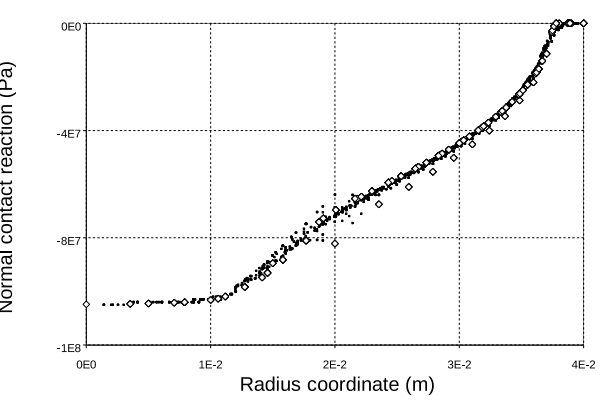

b

Figure 7. The normal contact reaction $v$ s radius. Diamonds represent the FEM. Field squares represent the X-FEM for tetrahedra (a) and hexaedra (b) 
We first note the ability of the method to capture the transition between the adherent region at the centre, characterized by an almost uniform pressure and the sliding domain where $\lambda$ increases to reach a zero value when no contact is detected. The discrepancy with the FEM result remains small for the X-FEM hexaedra case. It increases for the X-FEM tetrahedra case, because of the reduction of the Lagrangian space as described in 3.2 in order to satisfy the LBB condition.

\section{Conclusion}

An X-FEM formulation for frictional interfaces with possible large sliding has been proposed. Contact and friction are described following the augmented Lagrangian approach of (Ben Dhia et al., 2002), but we replaced a fixed point on friction threshold by the corresponding linearized terms in the tangent operator. This improves the robustness and convergence of the global iterative algorithm, as suggested in (Alart et al., 1991). The X-FEM spatial discretization is inspired from (Béchet et al., 2009) with the displacement interpolation used for the contact and friction unknowns, but extended to 3D meshes composed of tetrahedra or hexaedra. Large sliding is accounted for like in (Nistor et al., 2009), with a contact element built as a mixed slave and and master enriched bulk element.

As proposed by (Béchet et al., 2009), to ensure the satisfaction of the LBB condition, the space of contact and friction unknowns is reduced thanks to an algorithm that selects vital edges which unknowns are linearly associated to the ones of non vital edges. The contact status of this vital edge is preeminent over the contact statuses of non vital edges associated to it, and priority rules were established in case of multiple choice for the vital edge: : the vital edge must support a contact point. This enables to solve error in the normal contact reaction coming from the transition between contacting and non contacting regions. The same choice can be applied to the transition between adherent and sliding regions, where we favour the vital edge to be adherent. A numerical example of the compression of a $3 \mathrm{D}$ cylinder cut along a radial section illustrates the robustness of the method.

\section{References}

Alart P., Curnier A., A mixed formulation for frictional contact problems prone to Newton like solution methods, Comp. Meth. Appl. Meth. Engng., vol. 92, p. 353-375, 1991.

Babus̃ka I., The finite element method with Lagrangian multipliers , Numerische Mathematik, vol. $20, \mathrm{n}^{\circ} 3$, p. $179-192,1973$.

Ben Dhia H., Zarroug M., Hybrid frictional contact particles-in elements , Revue Europénne des éléments Finis, vol. 11, p. 417-430, 2002.

Brezzi F., Fortin M., Mixed and hybrid finite element methods, Springer - Verlag, 1991.

Béchet E., Moës N., Wohlmuth B., A stable lagrange multiplier space for stiff interface conditions within the extended finite element method , Int. J. Numer. Meth. Engng., vol. 78, $\mathrm{n}^{\circ} 8$, p. 931-954, 2009. 
Chapelle D., Bathe K., The inf-sup test ,Computers \& Structures, vol. 47, n 4/5, p. 537-545, 1993.

Dolbow J., Moës N., Belytschko T., An extended finite element method for modeling crack growth with frictional contact, Computer Methods in Applied Mechanics and Engineering, vol. 190, p. 6825-6846, 2001.

Dumont G., Algorithme des contraintes actives et contact unilatéral sans frottement, Revue Européenne des Éléments Finis, vol. 4, n 1, p. 55-73, 2001.

Géniaut S., Massin P., Moës N., Fissuration avec X-FEM et contact , Actes du 7ème Colloque National en Calcul des Structures, Giens, 17-20 may, 2005.

Géniaut S., Massin P., Moës N., A stable 3D contact formulation for cracks using XFEM , Revue Européenne de Mécanique Numérique, vol. 16, n 2, p. 259-275, 2007.

Khoei A., Nikbakht M., Contact friction modeling with the extended finite element method (X-FEM), Journal of Materials Processing Technology, vol. 177, p. 58-62, 2006.

Liu F., Borja R., A Contact algorithm for frictional crack propagation with the extended finite element method, Int. J. Numer. Meth. Engng., vol. 76, p. 1489-1512, 2008.

Moës N., Béchet É., Tourbier M., Imposing Dirichlet boundary conditions in the extended finite element method, Int. J. Numer. Meth. Engng., vol. 67, n 12, p. 1641-1669, 2006.

Moës N., Dolbow J., Belytschko T., A finite element method for crack growth without remeshing , Int. J. Numer. Meth. Engng., vol. 46, p. 131-150, 1999.

Mourad H., Dolbow J., Harari I., A bubble-stabilized finite element method for Dirichlet constraints on embedded interfaces, Int. J. Numer. Meth. Engng., vol. 69, n 4, p. 772-793, 2007

Nistor I., Guiton M., Massin P., Moës N., Géniaut S., An X-FEM approach for large sliding contact along discontinuities, Int. J. Numer. Meth. Engng., vol. 78, n ${ }^{\circ} 12$, p. 1407-1435, 2009. 
